
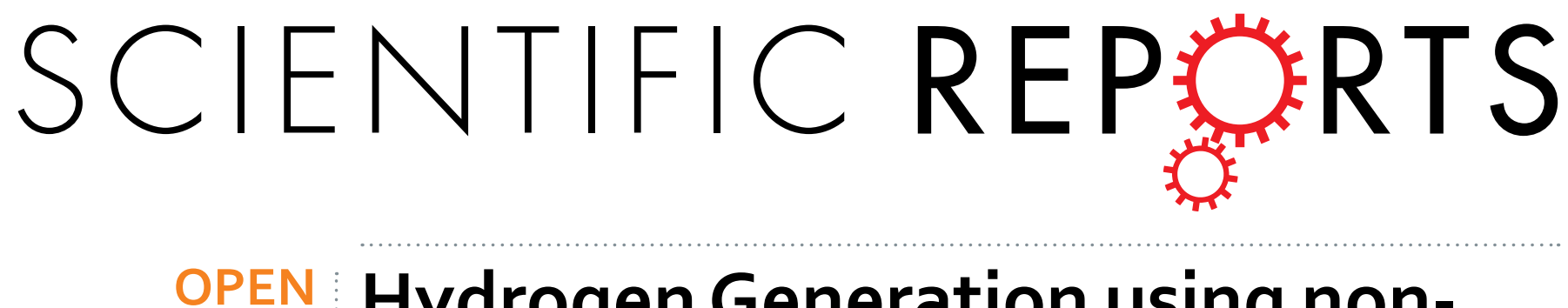

\title{
Hydrogen Generation using non- polar coaxial InGaN/GaN Multiple Quantum Well Structure Formed on \\ Hollow n-GaN Nanowires
}

Received: 19 May 2016

Accepted: 01 August 2016

Published: 24 August 2016
Ji-Hyeon Park ${ }^{1}$, Arjun Mandal ${ }^{1}$, San Kang
, Uddipta Chatterjee $^{1}$, Jin Soo Kim
Byung-Guon Park

This article demonstrates for the first time to the best of our knowledge, the merits of InGaN/GaN multiple quantum wells (MOWs) grown on hollow n-GaN nanowires (NWs) as a plausible alternative for stable photoelectrochemical water splitting and efficient hydrogen generation. These hollow nanowires are achieved by a growth method rather not by conventional etching process. Therefore this approach becomes simplistic yet most effective. We believe relatively low Ga flux during the selective area growth (SAG) aids the hollow nanowire to grow. To compare the optoelectronic properties, simultaneously solid nanowires are also studied. In this present communication, we exhibit that lower thermal conductivity of hollow $n-G a N ~ N W s$ affects the material quality of InGaN/GaN MOWs by limiting In diffusion. As a result of this improvement in material quality and structural properties, photocurrent

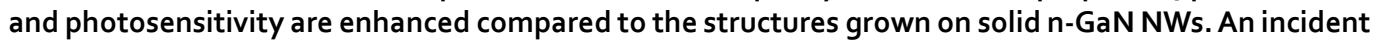
photon-to-current efficiency (IPCE) of around $\sim 33.3 \%$ is recorded at $365 \mathrm{~nm}$ wavelength for hollow NWs. We believe that multiple reflections of incident light inside the hollow $n$-GaN NWs assists in producing a larger amount of electron hole pairs in the active region. As a result the rate of hydrogen generation is also increased.

Over the past two decades, III-V compound semiconductor materials have been used extensively in the field of optoelectronics. In particular, various GaN-based device structures with outstanding features have been developed and realized for their significant applications as light emitting diodes (LEDs), lasers, solar cells, and photodetectors $^{1,2}$. Besides these applications, metal-nitride semiconductors have also emerged as a new generation of materials for the applications of photoelectrochemical (PEC) water splitting and hydrogen generation ${ }^{3-5}$. The effective capture of sunlight and subsequent conversion into chemical fuels such as hydrogen has attracted considerable attention ${ }^{6-12}$. Compared to solar electricity, the use of chemical bonds to store solar energy promises significantly reduced device fabrication cost, as well as the cost associated with energy storage ${ }^{13,14}$. In this regard, hydrogen production by photoelectrolysis of water with semiconductor materials offers a clean, environmentally friendly process ${ }^{13-15}$.

A semiconductor that is to be used as a water splitting photoelectrode must meet several requirements related to its band gap and electronic energies. It also needs to be sufficiently stable that it is not damaged by the water splitting environment ${ }^{16}$. Both $\mathrm{GaN}$ and InN are known for their chemical stability. The potential difference between the hydrogen-producing half-reaction and the oxygen-producing half-reaction is $1.229 \mathrm{~V}$. Thus, the band edges of InGaN (nearly $1.23 \mathrm{eV}$ ) can overlap the reduction and oxidation processes by providing the thermodynamically necessary energy to spilt water ${ }^{16}$. Moreover, among III-nitride semiconductors, InGaN is the only material whose energy bandgap can be tuned across nearly the entire solar spectrum ${ }^{17}$. In this study, we had dealt with In GaN/GaN multiple quantum wells (MQWs) structures grown on $\mathrm{n}-\mathrm{GaN}$ 1-D nanostructers for PEC water

${ }^{1}$ Semiconductor Materials Process Laboratory, School of Advanced Materials Engineering, Engineering College, Research Center for Advanced Materials Development (RCAMD), Chonbuk National University, Baekje-daero 567, Jeonju 54896, Republic of Korea. ${ }^{2}$ Department of physics, Chungnam National University, 220 Gung-dong, Yuseong-gu, Daejeon 34134, South Korea. ${ }^{3}$ Department of Polymer Nano Science and Technology, Chonbuk National University, Baekje-daero 567, Jeonju 54896, Republic of Korea. Correspondence and requests for materials should be addressed to C.-R.L. (email: crlee7@jbnu.ac.kr) 
splitting and hydrogen generation purpose. Also, GaN nanowires (NWs) offer a wide direct band gap (3.4 eV), high thermal conductivity $\left(1.3 \mathrm{Wcm}^{-1} \mathrm{~K}^{-1}\right)$, and highly saturated electron velocity, making themselves an obvious choice for improved device applications ${ }^{18-22}$.

PEC water splitting and hydrogen generation results using GaN based structures had been reported by many research groups. K. Maeda et al. reported $\mathrm{H}_{2}$ generation using a solid solution containing $\mathrm{GaN}$ and $\mathrm{ZnO}^{23}$. A detailed review of $\mathrm{GaN}$ and $\mathrm{ZnO}$ solid solution applied in water splitting can be found in ref. 24. Whereas K. Fujii et al. in 2005 showed n-type $\mathrm{GaN}$ as the working electrode for $\mathrm{H}_{2}$ generation using photoelectrochemical reactions ${ }^{3}$. In the same year Fujii and co-workers compared photoelectrochemical properties of p-type GaN with n-type GaN ${ }^{4}$. Very recently AlOtaibi et al. ${ }^{16}$ and Kibria et al. ${ }^{25}$ demonstrated water splitting and hydrogen generation using InGaN/ $\mathrm{GaN}$ core/shell NW-based heterostructures. These uniaxial heterostructures were grown using plasma-assisted molecular beam epitaxy (PAMBE) technique. Moreover, AlOtaibi et al. ${ }^{16}$ reported an incident photon-to-current efficiency (IPCE) of $\sim 27 \%$. But J. Kamimura et al. found IPCE around $40 \%{ }^{26}$. Whereas Kibira et al. reported an IQE of $60 \%$ by tuning the surface Fermi level of p-type $\mathrm{GaN}^{27}$. Furthermore J. Benton et al. ${ }^{28}$ and N. H. Alvi et al. ${ }^{29}$ reported IPCE of $46 \%$ and $67 \%$ respectively. In their review paper S. Zhao et al. extensively studied the evolution of IPCE in various $\mathrm{GaN}$ devices ${ }^{30}$. In this present scenario, to offer a polarization free larger effective area using $\mathrm{m}$-plane InGaN/GaN heterostructures for maximum light harvesting and efficient hydrogen generation, a new growth method was applied to demonstrate a simpler but sophisticated InGaN/GaN heterostructure. The structures presented in this report InGaN/GaN MQWs on n-GaN 1-D nanostructers. InGaN/ GaN MQWs were grown both on solid and hollow n-GaN NWs. InGaN/GaN MQWs grown on hollow n-GaN NWs were unique structures and an IPCE as high as $\sim 33.3 \%$ is being reported which is not the highest recorded number for nanostructures though it is the best reported IPEC value for a hollow NWs system without utilizing any external photocatalyst. n-GaN NWs, both solid and hollow, were grown on $\mathrm{Si}$ (111) substrates using PAMBE but coaxial InGaN/GaN MQWs were grown on $n-G a N$ NWs using metal organic chemical vapor deposition (MOCVD) process.

\section{Methodology}

Growth of Solid and Hollow n-GaN NWs. As mentioned earlier, a unique concept of hollow n-GaN NWs for efficient hydrogen generation was introduced in this study. Our approach was to make a comparative study between solid n-GaN NWs and hollow n-GaN NWs both grown on Si (111) substrates using PAMBE technique. To control the size and pitch of $\mathrm{n}-\mathrm{GaN}$ NWs during growth, we used nano-patterned Si (111) substrate. To initiate, polystyrene (PS) beads of diameter $200 \mathrm{~nm}$ were first diluted in deionized water and then spin coated on Si (111) substrate. These monolayer-thick PS beads were then reduced to a diameter of $80 \mathrm{~nm}$ by reactive ion etching process, treated under oxygen plasma. Next, Ti films $15 \mathrm{~nm}$ thick were deposited over the PS beads using electron-beam deposition and finally, PS beads were removed by lift-off using ultrasonic bath. Patterned Si (111) substrates were then introduced into the ultra-high vacuum growth chamber of the PAMBE system equipped with a rf-plasma source for providing active nitrogen and standard Knudsen cells for solid source Ga and n-type dopant silicon. Prior to the growth of both solid and hollow n-GaN NWs, the temperature of the Si substrate was stabilized at $600^{\circ} \mathrm{C}$ for ten minutes under nitrogen plasma atmosphere. Afterwards, Si-doped n-GaN NWs were grown on the patterned $\mathrm{Si}$ substrate at $750^{\circ} \mathrm{C}$. Growth recipes for both types of $\mathrm{n}-\mathrm{GaN}$ NWs retained the same growth parameters except in the Ga flux. The Ga flux pressure was maintained at $5.0 \times 10^{-7}$ torr and $2.5 \times 10^{-7}$ torr for growing solid and hollow n-GaN NWs respectively. But to enclose the top part of the hollow nanowire, we increased the Ga flux to $3.0 \times 10^{-7}$ torr for the last 10 minutes of growth process. Schematics representing the growth mechanism for solid and hollow n-GaN NWs are shown in Fig. 1. In case of the hollow NWs, the scarcity of $\mathrm{Ga}$ flux results $\mathrm{GaN}$ nucleation in periphery of the Ti masking pattern. After the initial edge nucleation interestingly the growth continues predominantly to be in the vertical direction instead of filling the patterned hole due to the higher activation energy of GaN than Ti. As also observed by F. Schuster et al. in their report, the less Ga presence in growth atmosphere should result into nanotubes by controlling the pattern density ${ }^{31}$. As shown in the Fig. 1, we increased Ga flux to close the top of the hollow NWs to avoid occurrence of stacking fault defect or any dislocation inside the nanohole while growing MQWs along the m-plane of the NWs. One can also observe some open hollow NWs or nanotubes in Fig. 1 because of limited Ga flux. On the other hand, affluent Ga flux results nucleation filling entire area of patterned hole which in turn reults into solid NWs.

Schematic representation of the experimental procedures for growing coaxial InGaN/GaN MQWs on solid and hollow n-GaN NWs where a crosssectional representation of InGaN/GaN MQWs are shown for two front hollow NWs.

Growth of Coaxial MQWs on Hollow and Solid n-GaN NWs. Messanvi et al. in 2015 have commented on better light harvesting and higher internal quantum efficicency of MQWs over thick quantum well structure ${ }^{32}$. Moreover, MQWs having a narrow barrier layer (less than $4 \mathrm{~nm}$ ) can demonstrate higher IQE due to carrier tunneling as reported by J. J. Wierer et al. in $2012^{33}$. So in the light of aforementioned findings, InGaN/GaN MQWs structures were designed further as active layers on PAMBE grown n-GaN NWs using MOCVD to find out how a through vacuum space inside a hollow n-GaN NW tunes the material and electrical properties of the devices. In the MOCVD system, nine pairs of InGaN/GaN MQWs were subsequently grown on n-GaN NWs using trimethylgallium (TMGa), trimethylindium (TMIn) and $\mathrm{NH}_{3}$ as the $\mathrm{Ga}$, In, and $\mathrm{N}$ sources, respectively. Hydrogen gas was used as a carrier gas. The growth pressure was maintained at 400 Torr. We utilized the pulsed flow precursor method to grow the MQWs structures of InGaN wells and GaN barriers. Each InGaN well was grown at $640^{\circ} \mathrm{C}$ for 200 seconds whereas $\mathrm{GaN}$ barrier was grown at $780^{\circ} \mathrm{C}$ for 180 seconds. Finally, the n-GaN capping layer was grown for 10 minutes at $800^{\circ} \mathrm{C}$. Silane $\left(\mathrm{SiH}_{4}\right)$ was used for $\mathrm{n}$-type doping. During the growth, the flow rates of $\mathrm{Ga}, \mathrm{NH}_{3}, \mathrm{SiH}_{4}$, and In were maintained at $0.3 \mathrm{sccm}, 3 \mathrm{slm}, 10 \mathrm{sccm}$, and $10 \mathrm{sccm}$, respectively. The thickness of GaN barrier layer was kept less than $4 \mathrm{~nm}$ whereas InGaN well was grown to be less than $3 \mathrm{~nm}$ 


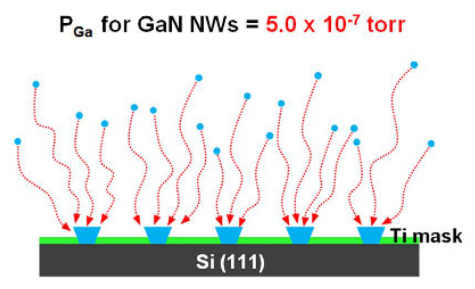

Nuclation of $n-G a N$ nanowire

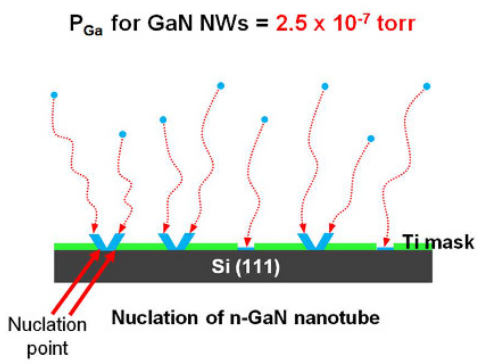

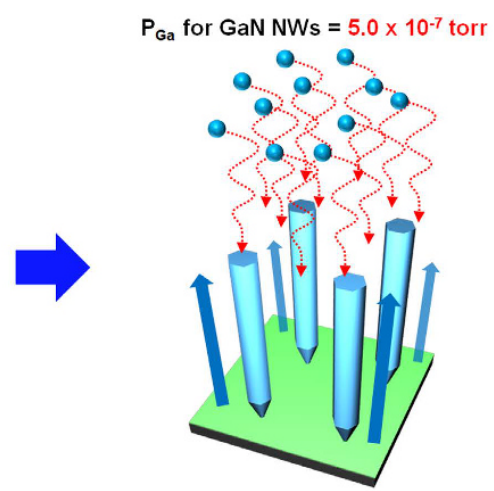

Solid n-GaN NWs

$P_{\text {Ga }}$ for GaN NWs $=3.0 \times 10^{-7}$ torr

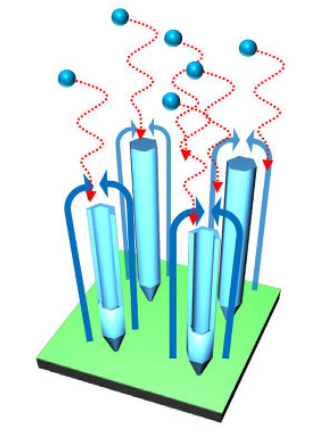

Hollow n-GaN NWs

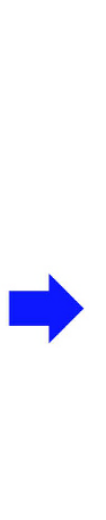

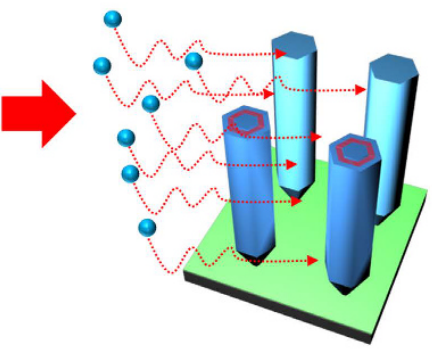

InGaN/GaN 9 pairs MQW on solid $n-G a N$ NWs
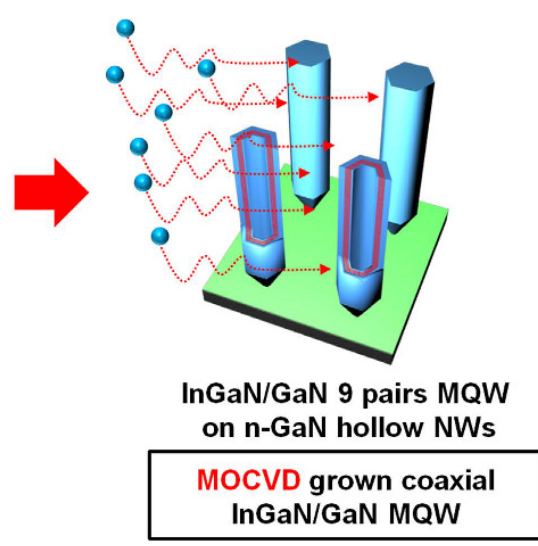

Figure 1. Schematic representation of the growth mechanism for both hollow and solid NWs. Schematic representation of the experimental procedures for growing coaxial InGaN/GaN MQWs on solid and hollow $\mathrm{n}-\mathrm{GaN}$ NWs where a crosssectional representation of InGaN/GaN MQWs are shown for two front hollow NWs.

for both the types of NWs. Schematic representations of the whole quantum structure for both the types of NWs are shown in Fig. 1.

Optical Properties and PEC Measurements. Au/Ni metal electrodes were fabricated on both types of samples consisting of solid and hollow $\mathrm{n}-\mathrm{GaN}$ NWs for photocurrent measurements. In order to record the PEC measurements, a three electrode system was used: a sample was used as the working electrode, Pt mesh as counter electrode, and $\mathrm{Ag} / \mathrm{AgCl}(1 \mathrm{M} \mathrm{KCl})$ as reference electrode. Incident light with a power density of $100 \mathrm{~mW} / \mathrm{cm}^{2}$ was used as a light source during the water splitting experiment. PEC measurements were carried out in a homemade quartz chamber at room temperature. Before the measurement, deionized water was purged into the setup using Ar for 40 minutes. Using a thermal conductivity detector gas chromatography (TCD GC) system (Agilent-6890N), the amount of the evolved $\mathrm{H}_{2}$ was measured. Sampling was carried out using a vacuum syringe. High purity Ar (99.99999\%) was used as a carrier gas.

Characterization. A Hitachi S-7400 system was used for the FE-SEM study; it was operated at $15 \mathrm{kV}$ and at a $13^{\circ}$ tilt-view. A JEOL JEM-ARM-200F system operated at $200 \mathrm{KV}$ was used in the HR-TEM study. Samples of the NW structures were prepared by coating with Platinum using a dual-beam focused ion beam (FIB, Quanta 3D FEG) technique with a beam current of $65 \mathrm{nA}$ and a resolution of $7 \mathrm{~nm}$ at $30 \mathrm{KV}$. Single-crystal X-ray diffraction (XRD) measurements were performed using a Rigaku diffractometer equipped with a $\mathrm{Cu}-\mathrm{K} \alpha$ radiation source. PL spectroscopy was carried out using a $325 \mathrm{~nm}$ line of a He-Cd laser as an excitation source at room temerature. CL measurements were taken at the applied accelerating voltage $\left(\mathrm{V}_{\mathrm{a}}\right)$ and beam current $\left(\mathrm{I}_{\mathrm{b}}\right) 10 \mathrm{KV}$ and $1000 \mathrm{pA}$, respectively. For measuring photocurrent of the fabricated devices, we utilized a solar simulator (McScience Lab 100 ) as a light source. This light source generated white light with a maximum power density of $100 \mathrm{~mW} / \mathrm{cm}^{2}$. A monochromator (Oriel Cornerstone 130) was used to provide the monochromatic light incident on the channel. 

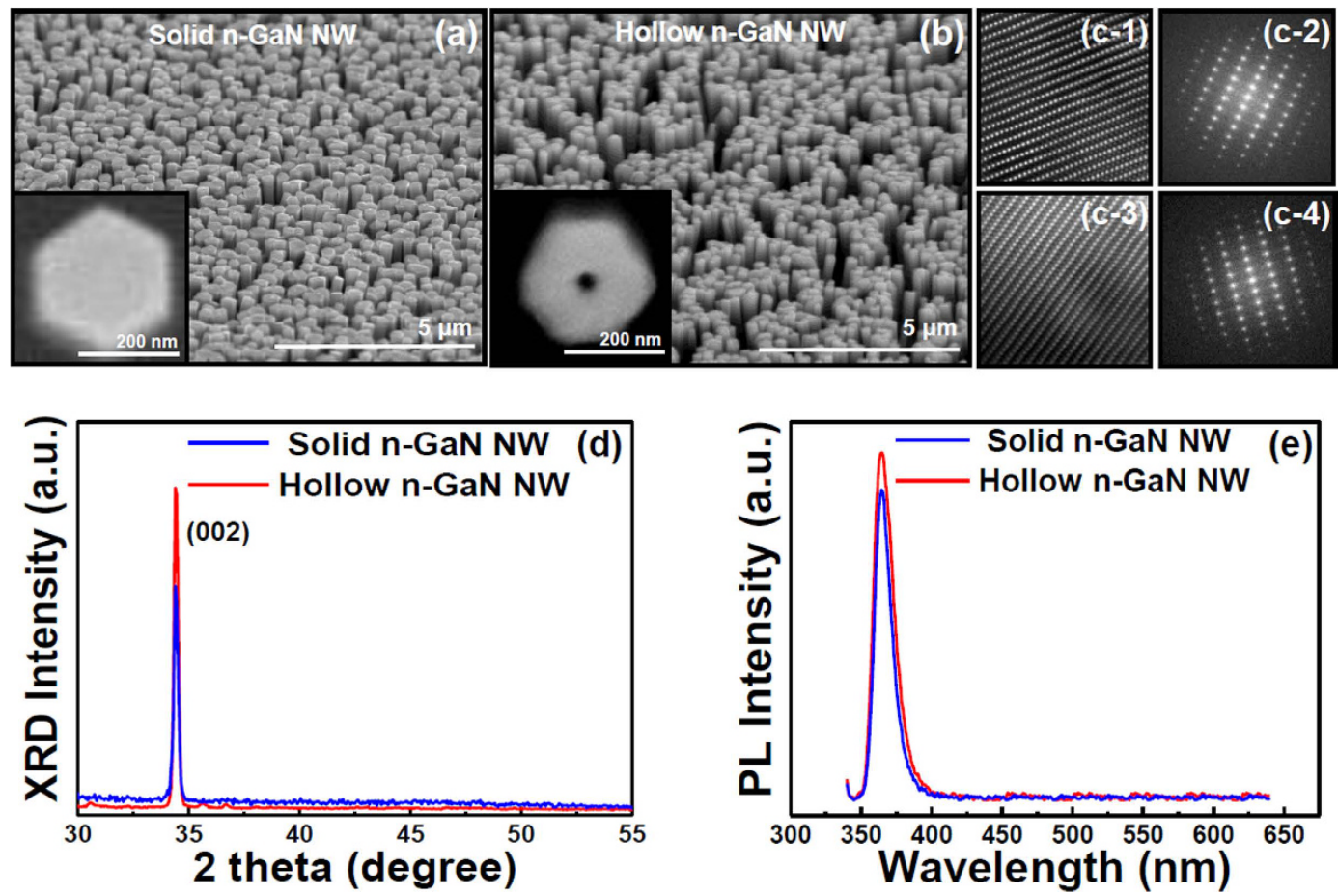

Figure 2. Tilt view FE-SEM images of (a) solid n-GaN NWs, (b) hollow n-GaN NWs; top view FE-SEM images of (a inset) solid n-GaN NW shows clear hexagonal shape and (b inset) a nano hole was noticed at the center of hexagonal hollow $n-G a N ~ N W ;(c-1,3)$ and $(\mathbf{c}-\mathbf{2}, \mathbf{4})$ show IFFT and SAED patterns of the solid and hollow n-GaN NW, respectively; (d) single-crystal XRD patterns of solid and hollow n-GaN NWs; (e) comparison of room temperature PL spectra of solid and hollow n-GaN NWs.

\section{Results and Discussion}

Structural Comparison between Solid and Hollow NWs. Figure 2a,b show tilt view field emission scanning electron microscopy (FE-SEM) images of solid and hollow n-GaN NWs, respectively, after grown with PAMBE. As the Ga flux was made half during the growth of hollow NWs, their density also became less (Fig. 2b) compared to the solid n-GaN NWs (Fig. 2a). High resolution FE-SEM images of a single solid and hollow n-GaN NWs were shown in Fig. 2a,b, respectively. A nano sized hole ( less than $40 \mathrm{~nm}$ ) at the center of the hexagonal hollow $\mathrm{n}-\mathrm{GaN}$ NW was noticed (Fig. $2 \mathrm{~b}$ inset). This inset image was taken after performing a FIB milling step which cut the $n-G a N$ hollow NWs from the middle exposing the nanohole. The morphology of the n-GaN NWs was further revealed by high-resolution transmission electron microscopy (HR-TEM). The Inverse Fast Fourier Transform (IFFT) imaging (Fig. 2c-1,3) proved the fact that both types of nanowires were free of defects and dislocations and they were well-grown hexagonal structures as the representative selected-area electron diffraction (SAED) pattern shown in Fig. 2c-2,4 respectively for solid and hollow structures. Moreover, X-ray diffraction investigation was carried on the grown NWs to study the crystal quality. X-ray diffraction pattern showed a strong presence of the (002) plane for both solid and hollow n-GaN NWs confirming good crystallinity and material quality of both types of $n-G a N ~ N W s$ (Fig. 2d). The XRD results matched those of GaN listed in the Joint Committee on Powder Diffraction Standards (JCPDS) cards No. 50-0792. Therefore, this can be confirmed that there was no difference in the material quality of solid and hollow n-GaN NWs after they were grown with different rates of Ga flux using PAMBE. Room temperature photoluminescence (PL) measurements also proved the fact. There was almost no difference in PL emissions from solid and hollow n-GaN NWs (Fig. 2e). With these revelations, it would be fascinating to study further the effects of vacuum existent in hollow n-GaN NWs on the active layer's material properties grown on NW body.

InGaN/GaN MQWs were grown on both solid and hollow n-GaN NWs using MOCVD and cross-sectional FE-SEM was further carried out. There was almost no difference noticed for both types of n-GaN NWs consisting of MQWs as active regions, from a morphological point of view (Fig. 3a,b). Figure 3a-1,b-1 show the FE-SEM images of a single solid and hollow $n-G a N ~ N W$, respectively with InGaN/GaN MQWs grown on the NW body. Figure 3a-2,b-2 present their respective cathodoluminescence (CL) mapping. We believe that different CL mapping for InGaN/GaN MQWs was caused by difference in thermal conductivity between hollow and solid nanowire (Fig. 3a-2,b-2, respectively) ${ }^{34}$. In the aforementioned report, the investigators demonstrated a reduction in thermal conductivity by $33 \%$ by introducing a small hollow area of $1 \%$ of the total size of a nanowire. In lieu with their conclusion, we also expect a smaller thermal conductivy in case of grown hollow nanowires. Thus, lower In outdiffusion occurs as a result of lower thermal conductivy which in turn enhances InGaN/GaN MQWs crystal quality in hollow nanowires. In presence can be clearly noticed at the bottom, middle, and top part of both the NWs from CL mapping (Fig. 3a-2,b-2). But noticeably CL mapping intensity is more in case of hollow nanowire as shown in the images caused by the lower In outdiffusion. Furthermore, room temperature 

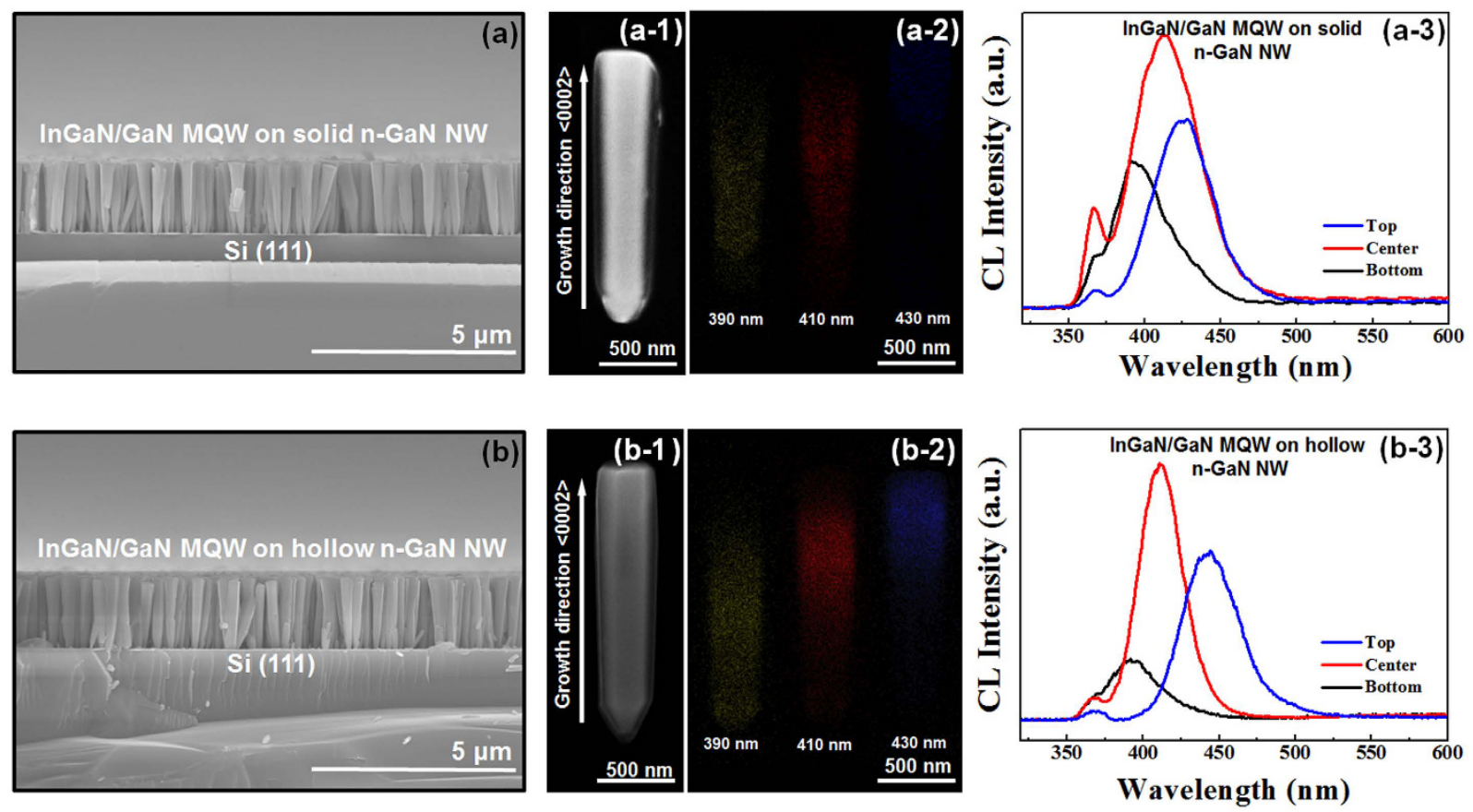

Figure 3. Cross sectional FE-SEM images of InGaN/GaN MQWs grown on (a) solid n-GaN NWs and (b) hollow n-GaN NWs; (a-1) and (b-1) show the FE-SEM images of single solid and hollow n-GaN NW, respectively, consisting of InGaN/GaN MQWs as active layers; (a-2) and (b-2) represent CL mappings of solid and hollow n-GaN NW, respectively at 390, 410 and $430 \mathrm{~nm}$ wavelength; (a-3) and (b-3) depict room temperature CL spectra of InGaN/GaN MQWs grown on solid and hollow n-GaN NWs, respectively.
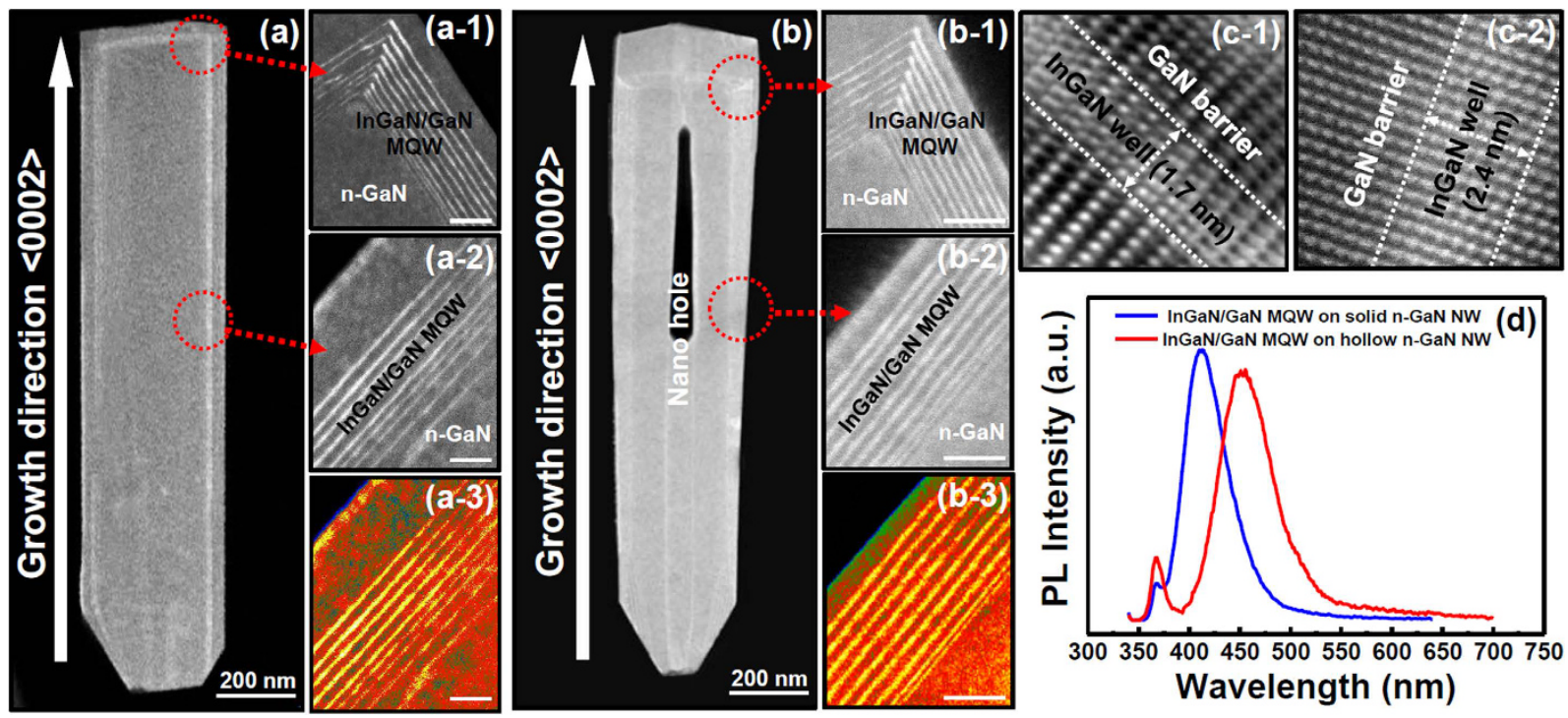

Figure 4. HR-TEM image of coaxially grown InGaN/GaN MQWs on (a) solid n-GaN NW and (b) hollow n-GaN NW; hollow $n-G a N ~ N W$ has a vacuum nano hole region inside; (a-1 2) and (b-1 2) show high magnification HR-TEM images of 9 pairs of InGaN/GaN MQWs grown on solid and hollow n-GaN NWs, respectively; (a-3) and (b-3) are the color representations of (a-2) and (b-2), respectively; (c-1) and (c-2) represent IFFT images of InGaN/GaN MQW grown on solid and hollow n-GaN NWs, respectively; (d) blue shift was noticed for the room temperature PL emission of InGaN/GaN MQWs grown on solid n-GaN NWs.

CL emissions of middle area show a broader full width at half-maximum (FWHM) of CL peak for InGaN/GaN MQWs grown on solid n-GaN NWs (Fig. 3a-3) compared to MQWs grown on hollow n-GaN NWs (Fig. 3b-3) which attributed to better material quality of InGaN/GaN MQWs when grown on hollow n-GaN NWs. More crystalline property studies were shown in TEM data. Visibly only the middle part of both the nanowires which is $\mathrm{m}$-plane, reveals better crystalline properties compared to top and bottom part because this area is mostly free of 
defects and strain as defects and strain are concentrated at the edges or corners of $\mathrm{c}$ and r-plane of nanowires ${ }^{35,36}$. Interestingly the top area CL emission of hollow nanowire shows a redshift to higher wavelength which is again demonstrated in PL data. This red shift likewise endorsed the uniform presence of In for hollow nanowires due to lower outdiffusion ${ }^{37}$.

Figure 4a shows the HR-TEM image of a single solid n-GaN NW with InGaN/GaN MQWs grown on it as an active region, whereas Fig. $4 \mathrm{~b}$ shows that of a single hollow n-GaN NW. Upon carefull inspection, one can notice the nano hole present throughout the length of nanowire in Fig. 4b. HR-TEM images give a closer look of the nine pairs of InGaN/GaN MQWs as Fig. 4a-1,2 and 3 show when grown on solid n-GaN NW and Fig. 4b-1,2 and 3 show when realized on hollow $n-G a N ~ N W$. If the colored HR-TEM images are compared for InGaN/GaN MQWs grown on solid and hollow $\mathrm{n}-\mathrm{GaN}$ NWs, one can observe that m-plane of the bottom layers of InGaN wells are not properly formed when grown on solid n-GaN NWs (Fig. 4a-3) whereas InGaN wells grown on hollow n-GaN NW exhibits nine pairs of well defined InGaN/GaN MQWs (Fig. 4b-3). We propose in this article that in the case of hollow $\mathrm{n}-\mathrm{GaN}$ NWs, there is a vacuum nano hole created inside (Fig. 4b) and this nano hole plays an important role in determining the structural and material qualities of the InGaN/GaN MQWs grown as active layers on the hollow NW body. Due to this nano hole the thermal conductivity gets lowered in hollow nanowire which ultimately decreases the In outdiffusion during the growth of InGaN/GaN MQWs ${ }^{34}$. When GaN barriers are grown over InGaN wells at a comparatively higher temperature, there is a major possibility of In-Ga intermixing ${ }^{34}$ and, thus, deformation of InGaN wells. When the high resolution IFFT images were compared for InGaN/GaN MQWs grown on both solid and hollow $\mathrm{n}-\mathrm{GaN}$ NWs, the phenomenon of In-Ga intermixing was clearly noticed for solid n-GaN NWs (Fig. 4c-1) and the width of the well was decreased to around $1.7 \mathrm{~nm}$ due to deformation. Figure 4c-2 showed the IFFT image of InGaN well and GaN barrier with almost no In-Ga intermixing and the width of InGaN well being around $2.4 \mathrm{~nm}$ when grown on hollow $\mathrm{n}$-GaN NW because of the lower In outdiffusion. GaN barrier layers were measured to be $4.5 \mathrm{~nm}$ and $3.8 \mathrm{~nm}$ for solid and hollow NWs respectively. Lower In-Ga, intermixing also caused a red shift in the room temperature PL emission of InGaN/GaN MQWs when grown on hollow n-GaN NWs compared to solid NWs (Fig. 4d) which is consistant with abovementioned CL spectrum. HR-TEM results confirmed the fact that the nano hole inside the hollow n-GaN NW reduced the In outdiffusion by lowering the thermal conductivity of InGaN/GaN MQWs and thus improving their material and structural properties. Figure $4(\mathrm{a} 1-3, \mathrm{~b} 1-3)$ were taken under $50 \mathrm{~nm}$ scale bar.

When light incidents on the InGaN/GaN MQWs grown on solid n-GaN NWs, the transition of carriers from the valence band to the conduction band happens throughout the heterostructure, from the surface n-GaN capping to the core $\mathrm{n}-\mathrm{GaN}$ NW. The electron hole pairs are generated in every layer and the band diagram of the heterostructure containing solid n-GaN NW is shown schematically in Fig. 5a. Also, the fraction of the incident light absorbed within the heterostructure does not experience any further multiple reflections while traversing through the solid n-GaN NW (Fig. 5a). The excited carriers in the conduction band of solid n-GaN NW core travel a long distance in both ways before they are absorbed inside the InGaN wells on either side and then take part in radiative recombination (Fig. 5a). Thus, there remains a possibility that these carriers may get absorbed within the defects and non-radiative recombination centers of the solid n-GaN NW core itself before they reach the InGaN well. Whereas in case of InGaN/GaN MQWs grown on hollow $n-G a N ~ N W s$, the transition of carriers from the valence band to the conduction band happens from the surface $n-G a N$ capping to the nano hole on either side when light falls on the heterostructure (Fig. 5b). The fraction of incident light absorbed within the nano hole undergoes multiple reflections as shown in Fig. 5b. When the multiplied reflected light from the nano hole is absorbed by the coaxially grown InGaN wells on either side, the rate of electron hole pair generation is also multiplied. The models we have proposed in Fig. 5 explains why the InGaN/GaN MQWs grown on hollow n-GaN NWs can produce more photo-generated carriers compared to those grown on solid n-GaN NWs. How the generation of electron hole pairs is helping in the evolution of hydrogen during PEC water splitting experiment is also examplified in Fig. 5. This model is also reorted by Z. Lian et al. in $2015^{38}$.

Optical Studies of Fabricated InGaN/GaN Heterostructures. Figure 6a shows the comparison of photocurrents at a bias of $-1.5 \mathrm{~V}$, measured from InGaN/GaN MQWs grown on both solid and hollow n-GaN NWs. The photocurrent measured from the device consisting of solid n-GaN NWs represented a flat band nature of photocurrent value around $0.01 \mathrm{~A}$ within the wavelength of 350 to $475 \mathrm{~nm}$. The photocurrent behavior of these coaxial InGaN/GaN MQWs are relatively better compared to the uniaxially grown InGaN/GaN core/shell NW based heterostructures ${ }^{15,22}$ as coaxially grown InGaN/GaN MQWs offer a larger active area. There was a fall in photocurrent value at around $500 \mathrm{~nm}$ wavelength which we believed was due to the limitation of the absorption range of the InGaN material we had grown (Fig. 6a). These deformed structures might take part in photocurrent generation. It was quite obvious that the improvement of the material quality of the InGaN/GaN MQWs grown on hollow n-GaN NWs, as concluded from the CL and HR-TEM results, helped in enhancing the photocurrent values compared to the wells grown on solid n-GaN NWs (Fig. 6a). Also, the participation of multiplied reflected light inside the nano hole enhanced photo carrier generation in case of MQWs grown on hollow n-GaN NWs (Fig. 5b). The photocurrent behavior also shows an increment around the wavelength $365 \mathrm{~nm}$ which can be attributed to hollow n-GaN core (Fig. 6a). Another increment can be seen around the wavelength $450 \mathrm{~nm}$ which we believe, is a result of photocurrent generated due the absorption by the $\mathrm{InGaN}$ well. Photocurrent results again confirmed the fact of the prominent existence of InGaN wells when grown on hollow $\mathrm{n}$-GaN NWs.

When photosensitivity ${ }^{39}\left(\mathrm{I}_{\mathrm{P}} / \mathrm{I}_{\text {dark }}\right)$ was recorded against applied bias at different wavelengths, photosensitivity values were found much higher for InGaN/GaN MQWs grown on hollow n-GaN NWs (Fig. 6c) compared to the wells grown on solid n-GaN NWs (Fig. 6b). Proper reasoning behind these phenomena goes with that of photocurrent already explained earlier in this article. Moreover, we measured the IPCE for these devices to further investigate the wavelength-dependent PEC properties of InGaN wells. The trend of IPCE was found likewise as in case of the photocurrent for InGaN/GaN MQWs grown on both solid and hollow $n-G a N$ NWs (Fig. 7). IPCE 

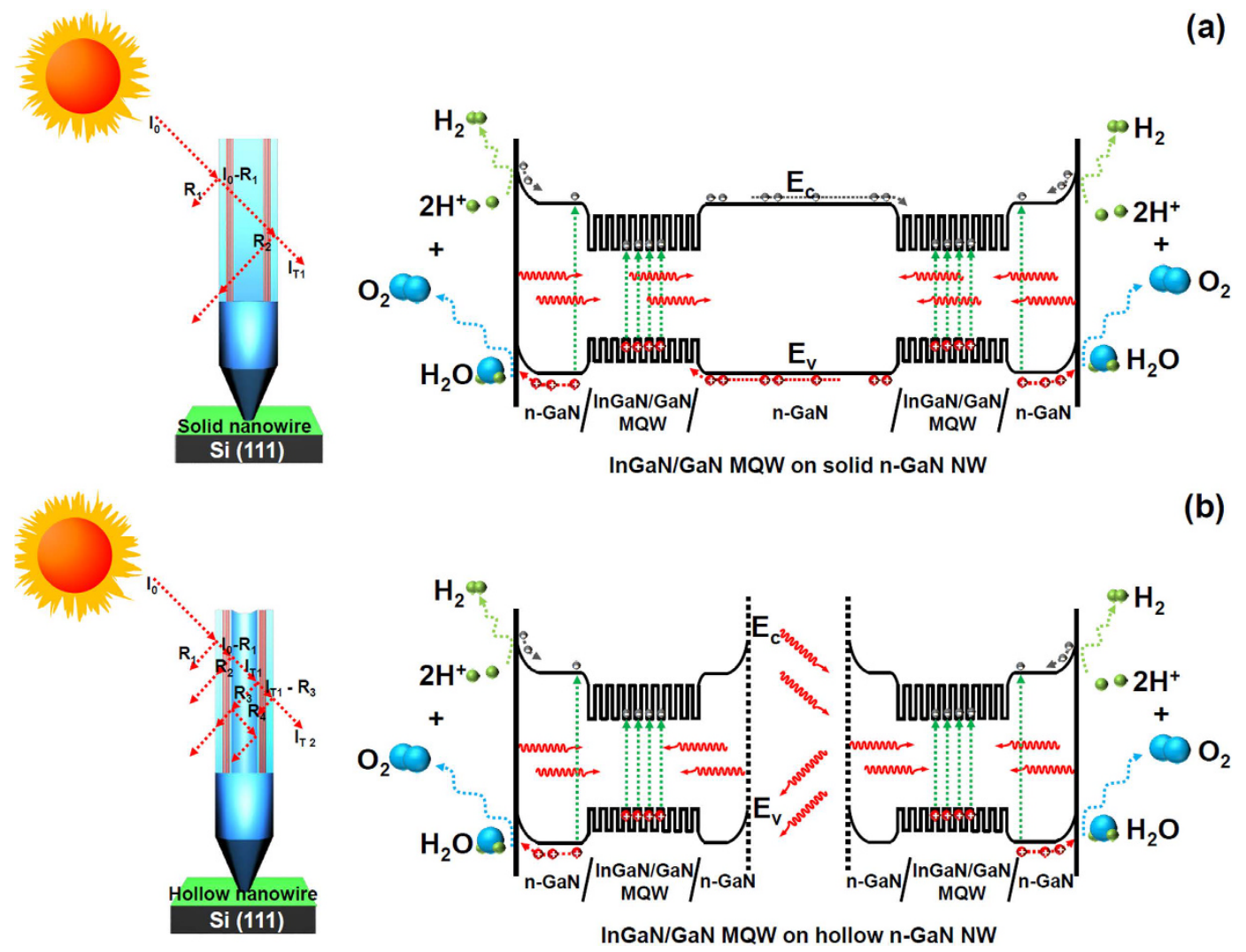

Figure 5. Schematic models to propose the reflection and absorption of incident light and electron hole pair generation inside the InGaN/GaN MQWs grown on (a) solid and (b) hollow n-GaN NWs. Band diagrams of both kinds of heterostructures are also presented to show how electron hole pair generation helps in hydrogen gas evolution in PEC water splitting experiment.

measured for MQWs grown on hollow $\mathrm{n}-\mathrm{GaN}$ NWs was about $33.3 \%$ at $365 \mathrm{~nm}$ wavelength. IPCE values also showed a sudden drop to $1.23 \%$ at $500 \mathrm{~nm}$ wavelength for MQWs grown on solid n-GaN NWs which was again due to the limitation of the absorption range of the InGaN material used. Photoresponses for both the type of devices gradually decrease after $500 \mathrm{~nm}$ wavelength. As also can be seen in Fig. 7, hollow $\mathrm{n}-\mathrm{GaN}$ NWs show a higher IPCE value than the solid NWs. This IPEC investigation also confirms that the hollow NWs are better at photo harvesting compared to the solid NWs which is consistent with our previous results.

PEC Studies on Fabricated InGaN/GaN Heterostructures. A schematic illustration of PEC experimental setup is shown in Fig. 8a. Figure 8b shows the measured current density as a function of applied potential for the InGaN/GaN MQWs grown on solid and hollow $\mathrm{n}-\mathrm{GaN}$ NWs under illumination conditions with a xenon arc lamp $(300 \mathrm{~W})$. The bias was applied to the $\mathrm{n}-\mathrm{GaN}$ working photoelectrode versus the Pt counter electrode, and measured from the $\mathrm{Ag} / \mathrm{AgCl}$ reference electrode. The behavioral trend of the current is similar to that of photocurrents obtained from InGaN/GaN MQWs grown on both solid and hollow n-GaN NWs (Fig. 6a). In both cases, there was almost a four-fold increase in current for the wells grown on hollow n-GaN NWs compared to that grown on solid n-GaN NWs. Only an overall decrease in current density value was noticed due to the higher refractive index of water if compared with Fig. $6 \mathrm{a}$. Around an applied bias of $-1.5 \mathrm{~V}$, a turn on and steep increase of current density were observed for the MQWs grown on hollow n-GaN NWs (Fig. 8b). This behavior of the biased channel can be correlated with the enhancement of the photosensitivity of the same structure at an applied bias of $-1.2 \mathrm{~V}$ (Fig. 6c). During the PEC water splitting and hydrogen generation experiment, ideally there should not be any applied voltage as the band edges of InGaN wells can straddle the oxidation and reduction potentials of water. To get rid of external losses such as the resistive loss of the system, a bias of $-1.5 \mathrm{~V}$ was applied in this experiment. A steady photocurrent was observed over a period of around two hours for both types of samples. Almost a 10 times increase of photocurrent was observed for the InGaN/GaN MQWs grown on hollow n-GaN NWs (Fig. 8d) compared to solid n-GaN NWs (Fig. 8c). The evolution of hydrogen gas from the Pt counter electrode over a period of one hour is also shown in Fig. $8 \mathrm{c}, \mathrm{d}$ for respective sampling. How the hydrogen is being evolved photo-chemically is already represented in Fig. 5a,b. The gas generation rate is directly proportional to the photocurrent density. With better material properties and improved electrical responses, it was expected that the hydrogen generation rate would be higher for MQWs grown on hollow n-GaN NWs if compared with solid n-GaN NWs. After one hour, around $415 \mu \mathrm{mol}$ hydrogen gas was generated by the former (Fig. $8 \mathrm{~d}$ ), whereas around $95 \mu \mathrm{mol}$ hydrogen was generated by the latter (Fig. $8 \mathrm{c}$ ). 

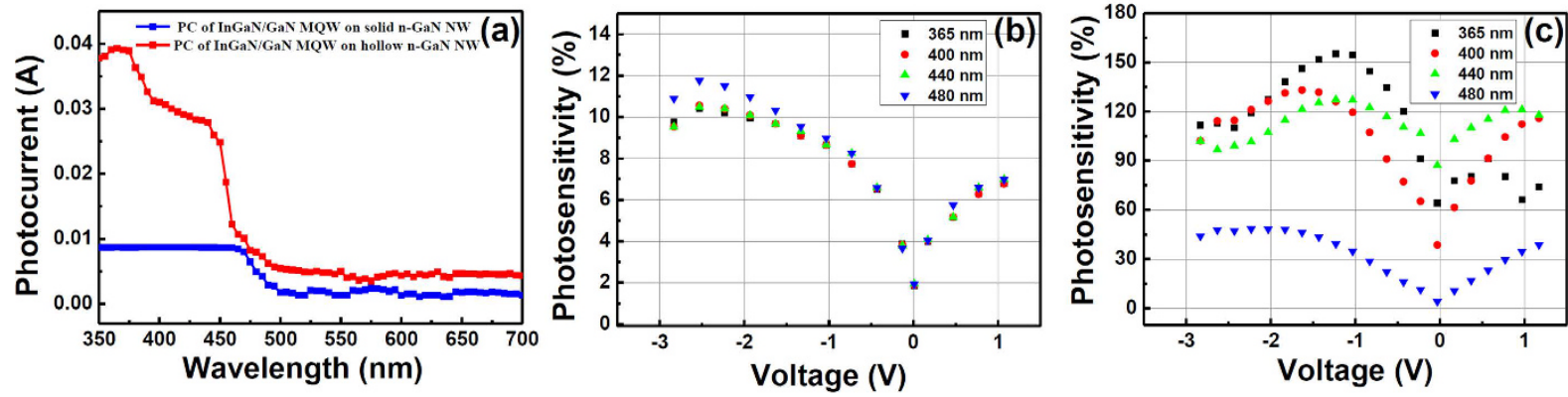

Figure 6. (a) Comparison of photocurrent recorded from InGaN/GaN MQWs grown on solid and hollow $\mathrm{n}-\mathrm{GaN}$ NWs at an applied bias of $-1.5 \mathrm{~V} ;(\mathbf{b}, \mathbf{c})$ show photosensitivity calculated at different wavelengths for InGaN/GaN MQWs grown on solid and hollow n-GaN NWs, respectively.

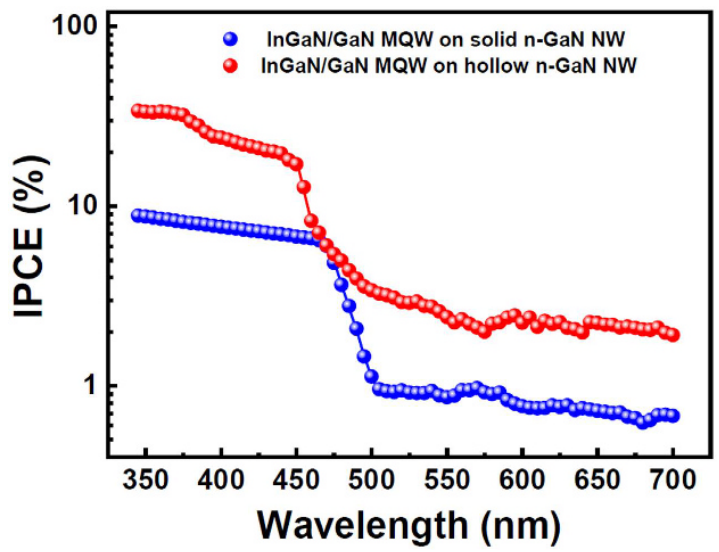

Figure 7. When IPCE data was compared for InGaN/GaN MQWs grown on both solid and hollow n-GaN NWs, a high IPCE value of $\sim 33.3 \%$ was observed for the later.
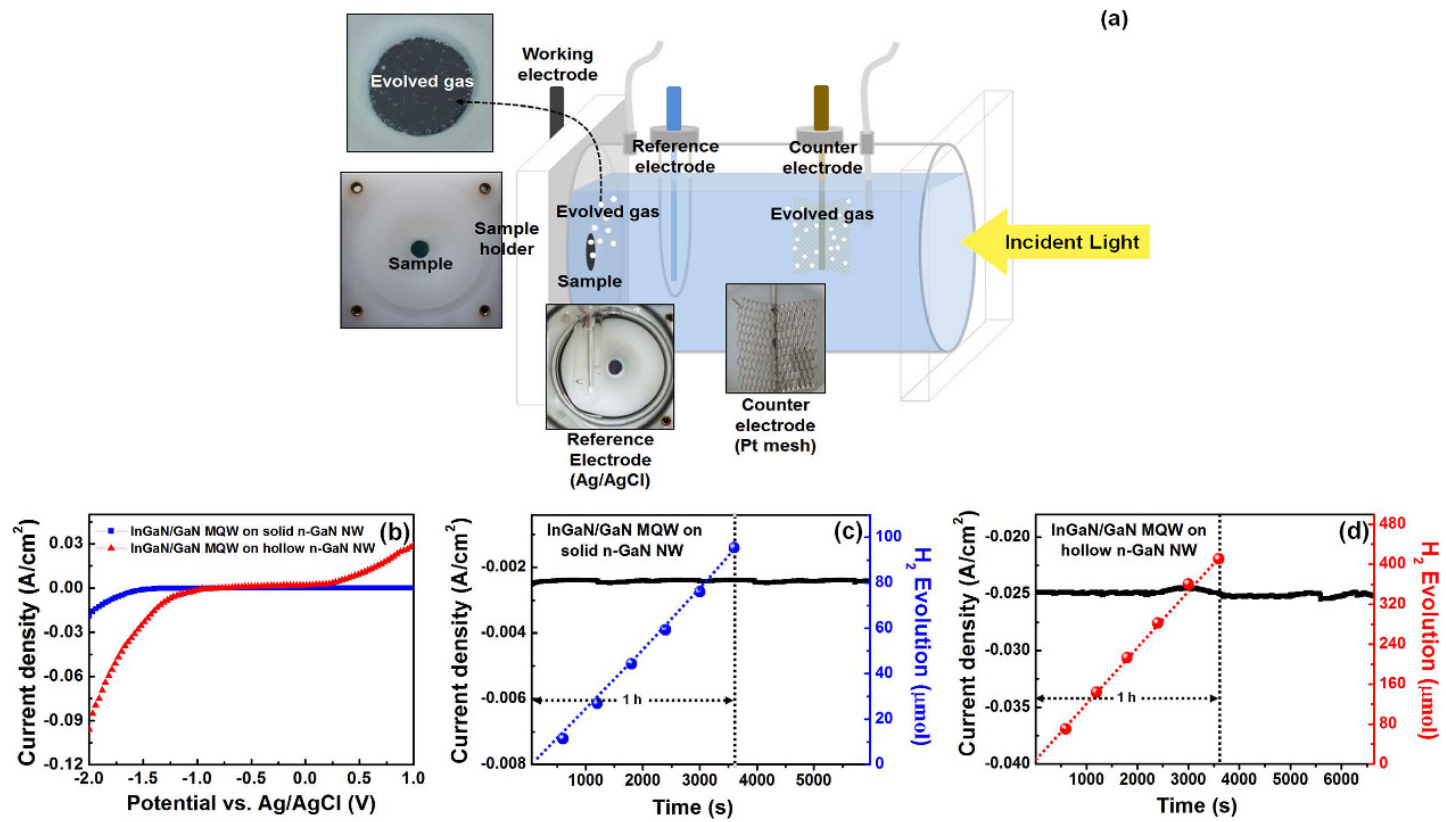

Figure 8. (a) Schematic of the PEC experimental setup, (b) Current density measured during PEC water splitting experiment from the InGaN/GaN MQWs grown on solid and hollow n-GaN NWs; at an applied bias of $-1.0 \mathrm{~V},(\mathbf{c}, \mathbf{d})$ show hydrogen evolution rate and current density of the InGaN/GaN MQWs grown on solid and hollow n-GaN NWs, respectively. PEC water splitting and hydrogen generation experiment was carried out under illumination with a $300 \mathrm{~W}$ xenon arc lamp. 


\section{Conclusion}

We report a new growth engineering where hollow $n-G a N$ NWs were grown using PAMBE. Furthermore to offer a polarization free larger active area InGaN/GaN MQWs was grown coaxially on the $n-G a N$ NWs using MOCVD. Moreover, excellent material properties were obtained for the MQWs grown on hollow n-GaN NWs. HR-TEM and CL studies proved the fact. Lower heat dissipation due to lower thermal conductivy in presence of nano hole inside the hollow $\mathrm{n}-\mathrm{GaN}$ NW controlled the In outdiffusion of InGaN/GaN MQWs structures resulting better material and structural properties. Multiple reflections of incident light inside the nano hole influenced more and more photo excitation and multiplication of electron hole pairs, resulting an enhanced photocurrent and a higher rate of hydrogen evolution. To conclude, an IPCE as high as $\sim 33.3 \%$ was recorded for the InGaN/ GaN MQWs grown on hollow n-GaN NWs which is the highest reported value for any hollow NWs structures without any external photocatalyst.

\section{References}

1. Bhattacharya, P. \& Mi, Z. Quantum-dot optoelectronic devices. Proc. IEEE 95, 1723-1740 (2007).

2. Schubert, E. F. Light-emitting diodes. Cambridge University Press. doi: 10.1109/PROC.1972.8592 (Cambridge University Press, 2010).

3. Fujii, K., Karasawa, T. \& Ohkawa, K. Hydrogen gas generation by splitting aqueous water using n-type GaN photoelectrode with anodic oxidation. Japanese J. Appl. Physics, Part 2 Lett. 44, 4-7 (2005).

4. Fujii, K. \& Ohkawa, K. Photoelectrochemical Properties of p-Type GaN in Comparison with n-Type GaN. Jpn. J. Appl. Phys. 44, L909-L911 (2005).

5. Theuwis, A., Strubbe, K., Depestel, L. M. \& Gomes, W. P. A Photoelectrochemical Study of InxGa1-xN Films. J. Electrochem. Soc. 149, E173 (2002).

6. G, C. The Photochemistry of the Future. Science (80-. ). 36, 385-394 (1912).

7. Fujishima, A. \& Honda, K. Electrochemical photolysis of water at a semiconductor electrode. Nature 238, 37-38 (1972).

8. Borgarello, E., Kiwi, J., Pelizzetti, E., Visca, M. \& Grätzel, M. Photochemical cleavage of water by photocatalysis. Nature 289, 158-160 (1981).

9. Khaselev, O. \& Turner, J. a. A Monolithic Photovoltaic-Photoelectrochemical Device for Hydrogen Production via Water Splitting R EPORTS A Monolithic Photovoltaic-Photoelectrochemical Device for Hydrogen Production via Water Splitting. Science (80-.). 280, 425-427 (1998).

10. Gratzel, M. Photoelectrochemical cells. Nat. (London, UK) 414, 338-344 (2001).

11. Maeda, K. \& Domen, K. Photocatalytic water splitting: Recent progress and future challenges. J. Phys. Chem. Lett. 1, 2655-2661 (2010).

12. Ebaid, M. et al. Enhanced solar hydrogen generation of high density, high aspect ratio, coaxial InGaN/GaN multi-quantum well nanowires. Nano Energy 12, 215-223 (2015).

13. Turner, J. a. A Realizable Renewable Energy Future. Science (80-. ). 285, 687-689 (1999).

14. Joshi, U. A., Palasyuk, A., Arney, D. \& Maggard, P. A. Semiconducting oxides to facilitate the conversion of solar energy to chemical fuels. J. Phys. Chem. Lett. 1, 2719-2726 (2010).

15. Trammell, S. A., Dressick, W. J., Melde, B. J. \& Moore, M. Photocurrents from the direct irradiation of a donor-acceptor complex contained in a thin film on indium tin oxide. J. Phys. Chem. C 115, 13446-13461 (2011).

16. AlOtaibi, B., Nguyen, H. P. T., Zhao, S., Kibria, M. G., Fan, S. \& Mi, Z. Highly Stable Photoelectrochemical Water Splitting and Hydrogen Generation Using a Double-Band InGaN/GaN Core/Shell Nanowire Photoanode. Nano Lett. 13, 4356-4361 (2013).

17. Wu, J. When group-III nitrides go infrared: New properties and perspectives. J. Appl. Phys. 106 (2009).

18. F. A., Ponce \& D. P. Bour. Nitride-based semiconductors for bluw and green light-emitting devices. Nature 386, 351-359 (1997).

19. Tang, Y. B. et al. Controllable synthesis of vertically aligned p-type GaN nanorod arrays on N-type Si substrates for heterojunction diodes. Adv. Funct. Mater. 18, 3515-3522 (2008).

20. Westover, T. et al. Photoluminescence, thermal transport and breakdown in Joule heated GaN nanowires. Nanoletters $\mathbf{9}, 257-263$ (2009).

21. Bertness, K. A. et al. Controlled nucleation of GaN nanowires grown with molecular beam epitaxy. Adv. Funct. Mater. 20, 2911-2915 (2010).

22. Chen, C. Y. et al. Gallium nitride nanowire based nanogenerators and light-emitting diodes. ACS Nano 6, 5687-5692 (2012).

23. Maeda, K. et al. GaN:ZnO solid solution as a photocatalyst for visible-light-driven overall water splitting. J. Am. Chem. Soc. 127, 8286-8287 (2005).

24. Adeli, B. \& Taghipour, F. A Review of Synthesis Techniques for Gallium-Zinc Oxynitride Solar-Activated Photocatalyst for Water Splitting. ECS J. Solid State Sci. Technol. 2, Q118-Q126 (2013).

25. Kibria, M. G. et al. Visible light-driven efficient overall water splitting using p-type metal-nitride nanowire arrays. Nat. Commun. 6, 6797 (2015).

26. Kamimura, J. et al. Photoelectrochemical properties of $(\mathrm{In}, \mathrm{Ga}) \mathrm{N}$ nanowires for water splitting investigated by in-situ electrochemical mass spectroscopy BT - Journal of the American Chemical Society. J. Am. Chem. Soc. 135, 10242-10245 (2013).

27. Kibria, M. G. et al. Tuning the surface Fermi level on p-type gallium nitride nanowires for efficient overall water splitting. Nat. Commun. 5, 1-6 (2014).

28. Benton, J., Bai, J. \& Wang, T. Utilisation of GaN and InGaN/GaN with nanoporous structures for water splitting. Appl. Phys. Lett. 105, 2012-2017 (2014).

29. Alvi, N. ul H. et al. InN/InGaN quantum dot photoelectrode: Efficient hydrogen generation by water splitting at zero voltage. Nano Energy 13, 291-297 (2015).

30. Zhao, S., Nguyen, H. P. T., Kibria, M. G. \& Mi, Z. III-Nitride nanowire optoelectronics. Prog. Quantum Electron. 44, 14-68 (2015).

31. Schuster, F. et al. Position-controlled growth of GaN nanowires and nanotubes on diamond by molecular beam epitaxy. Nano Lett. 15, 1773-1779 (2015).

32. Messanvi, A. et al. Investigation of Photovoltaic Properties of Single Core-Shell GaN/InGaN Wires. ACS Appl. Mater. Interfaces 7, 21898-21906 (2015).

33. Wierer, J. J., Koleske, D. D. \& Lee, S. R. Influence of barrier thickness on the performance of InGaN/GaN multiple quantum well solar cells. Appl. Phys. Lett. 100, 3-8 (2012).

34. Chen, J., Zhang, G. \& Li, B. Remarkable reduction of thermal conductivity in silicon nanotubes. Nano Lett. 10, 3978-3983 (2010).

35. Ra, Y.-H., Navamathavan, R., Kang, S. \& Lee, C.-R. Different characteristics of InGaN/GaN multiple quantum well heterostructures grown on $\mathrm{m}$ - and $\mathrm{r}$-planes of a single $\mathrm{n}-\mathrm{GaN}$ nanowire using metalorganic chemical vapor deposition. J. Mater. Chem. C 2, 2692 (2014).

36. Qian, F. et al. Controlled synthesis of AlN/GaN multiple quantum well nanowire structures and their optical properties. Nano Lett. 12, 3344-3350 (2012).

37. Tchernycheva, M. et al. InGaN/GaN Core - Shell Single Nanowire Light Emitting Diodes with Graphene-Based P - Contact. Nano Lett. 14, 2456-2465 (2014). 
38. Lian, Z. et al. Plasmonic silver quantum dots coupled with hierarchical TiO2 nanotube arrays photoelectrodes for efficient visiblelight photoelectrocatalytic hydrogen evolution. Sci. Rep. 5, 10461 (2015).

39. Ra, Y., Navamathavan, R., Park, J. \& Lee, C. Coaxial InxGa1-xN/GaN Multiple Quantum Well Nanowire Arrays on Si(111) Substrate for High-Performance Light-Emitting Diodes. Nano Lett. 13, 3506-3516 (2013).

\section{Acknowledgements}

This work was supported by the National Research Foundation of Korea (NRF) grant funded by the Korea government (MSIP) (No. 2015R1A4A1042417 and No. 2015R1A2A2A01002877).

\section{Author Contributions}

C.-R.L. planned and supervised the project; J.-S.K., B.-G.P. and M.-D. K. realized the hollow and solid type NWs using PAMBE, J.-H.P., A.M., S.K. and U.C. grew the MQWs using MOCVD on GaN NWs and performed the structural and PEC characterizations; J.-H.P. and A.M. analyzed the data; K.-U.J. helped into analyzing PEC characterization data; J.-H.P., A.M. and U.C. wrote the manuscript; all the authors discussed and commented on the manuscript.

\section{Additional Information}

Competing financial interests: The authors declare no competing financial interests.

How to cite this article: Park, J.-H. et al. Hydrogen Generation using non-polar coaxial InGaN/GaN Multiple Quantum Well Structure Formed on Hollow n-GaN Nanowires. Sci. Rep. 6, 31996; doi: 10.1038/srep31996 (2016).

(c) (i) This work is licensed under a Creative Commons Attribution 4.0 International License. The images or other third party material in this article are included in the article's Creative Commons license, unless indicated otherwise in the credit line; if the material is not included under the Creative Commons license, users will need to obtain permission from the license holder to reproduce the material. To view a copy of this license, visit http://creativecommons.org/licenses/by/4.0/

(C) The Author(s) 2016 